\title{
Efficacy of weekly paclitaxel treatment as a single agent chemotherapy following first-line cisplatin treatment in urothelial bladder cancer
}

\author{
SPYRIDON SIDERIS ${ }^{1}$, FOUAD AOUN ${ }^{2}$, MARC ZANATY ${ }^{3}$, NIEVES CHANZA MARTINEZ ${ }^{4}$, \\ SOFIA LATIFYAN ${ }^{1}$, AHMAD AWADA ${ }^{1}$ and THIERRY GIL ${ }^{1}$ \\ ${ }^{1}$ Medical Oncology Clinic; ${ }^{2}$ Department of Urology, Jules Bordet Institute, 1000 Brussels, Belgium; \\ ${ }^{3}$ Data Center, Université Paris XII, 94010 Créteil, France; ${ }^{4}$ Medical Oncology Clinic, \\ University Hospital of Erasme, 1070 Brussels, Belgium
}

Received September 7, 2015; Accepted March 3, 2016

DOI: $10.3892 / \operatorname{mco} .2016 .821$

\begin{abstract}
The aim of the present study was to investigate the efficacy of paclitaxel following a first-line cisplatin regimen in patients with metastatic bladder cancer. The present study retrospectively evaluated the clinical effects and toxicities of second-line paclitaxel regimens following first-line cisplatin treatment in metastatic bladder cancer. A total of 42 patients with progressing metastatic urothelial bladder cancer following cisplatin-based chemotherapy were enrolled. The patients received weekly treatment with paclitaxel $\left(80 \mathrm{mg} / \mathrm{m}^{2}\right)$ with a median duration of 3 months. The overall response rate, disease control rate and median progression free survival were 9.5, 45.2 and 6.4 months, respectively. Weekly paclitaxel was well-tolerated with rare grade III or IV toxicities. Second-line weekly paclitaxel treatment following first-line cisplatin-based chemotherapy is an effective and well-tolerated regimen in urothelial metastatic bladder cancer.
\end{abstract}

\section{Introduction}

Bladder carcinoma is the most common malignancy of the urinary tract and the ninth most common malignancy worldwide (1). In Europe it represents the fifth most common cancer type affecting predominantly men $>65$-years-old, usually smokers (2). Urothelial transitional cell carcinoma (TCC) accounts for $\sim 90 \%$ of all bladder cancer types.

In locally advanced or metastatic disease, platinum-based combination chemotherapy regimens are the standard of care as the first-line treatment (3). The combination of methotrexate, vinblastine, doxorubicin and cisplatin (MVAC) was the first

Correspondence to: Dr Fouad Aoun, Department of Urology, Jules Bordet Institute, Rue Heger-Bordet 1, 1000 Brussels, Belgium E-mail: fouad.aoun@bordet.be

Key words: paclitaxel, urothelial cancer, toxicity, overall survival, neutrophil to lymphocyte ratio regimen providing disease control and overall survival (OS) benefit (4), however, it is associated with several toxic effects, particularly when administered in a high-dose-intensity schema (5). A phase III trial demonstrated that compared with MVAC, the combination of gemcitabine and cisplatin (GC) resulted in similar objective response (49 vs. 36\%) and similar OS (14 vs. 15 months) (6) with a more favorable toxicity profile (7). The triple combination of paclitaxel and GC (PGC) is another option for patients with metastatic urothelial carcinoma. Compared with GC, PGC is associated with a significant improvement in the OS among patients with primary bladder cancer (median, 16 vs. 12 months; hazard ratio, 0.80; 95\% confidence intervals, 0.66-0.97), however, with increased incidence of serious grade III and IV toxicities (8). For patients with impaired renal function, carboplatin-based regimens can be used as the first-line for metastatic TCC, however, its efficacy remains to be evaluated with larger phase III trials (9). Non-platinum regimens, particularly those combining gemcitabine with either paclitaxel or docetaxel have also shown promising results in several phase II studies $(10,11)$.

Unfortunately, the majority of the patients with advanced or metastatic bladder cancer fail first-line chemotherapy in $<1$ year. A large proportion of these patients are not fit for second-line chemotherapy due to poor performance status, impaired renal function, advanced age and comorbidities. For those patients who are able to receive further treatment, no second-line regimen has been established. Chemotherapeutic agents, including vinflunine (12), pemetrexed (13), paclitaxel (14), docetaxel (15), gemcitabine (16) and ifosfamide (17), are used either as single agent regimens offering a response rate of a maximum of $20 \%$ or in combination providing improved response rates without necessarily improved OS, or with substantial cost in terms of toxicity (18-23).

Single agent paclitaxel is active in urothelial cancer. A phase II trial demonstrated a response rate of $42 \%$ in chemotherapy naïve patients with advanced TCC (24). In first-line treatment, the response rates ranged between 35 and $65 \%$, when combined with platinum agents (25). The experience with single agent paclitaxel following the failure of a platinum 
based regimen is limited and based upon small retrospective, or phase II studies.

The present study aimed to assess the response and toxicity rates of single agent paclitaxel in patients with metastatic urothelial cancer having progressed following a first-line cisplatin-based chemotherapy.

\section{Patients and methods}

The present study retrospectively evaluated 42 patients with metastatic urothelial bladder carcinoma treated with first-line cisplatin-based combination regimens and second-line paclitaxel monotherapy between January 2004 and January 2014 at the Jules Bordet Institute (Brussels, Belgium). The present study was approved by the Jules Bordet Institute Ethics Review Committee on the 18th December 2014. The histological diagnosis and staging of metastatic urothelial bladder carcinoma were based on the World Health Organization Classification and the TNM staging system, respectively. The eligibility criteria were histologically confirmed urothelial bladder cancer and metastatic stage treated by first-line cisplatin-based combination regimens and second-line single agent paclitaxel chemotherapy. Prior to initiating chemotherapy, each patient underwent physical examination, blood examinations, chest radiography, and thorax, abdomen and pelvis computed tomography. Brain and bone imaging were performed in the case of complaints. Pooled prospectively collected data were retrospectively analyzed. The optimal clinical response and maximum tumor shrinkage, according to the Response Evaluation Criteria In Solid Tumors (version 1.1), were considered as the tumor response. Complete response (CR) was defined as the disappearance of all target lesions, whereas partial response (PR) was a decrease in the sum of the target lesion diameters by at least $30 \%$ compared with the baseline. A progressive disease (PD) was considered if an increase of at least $20 \%$ occurred in the sum of the target lesion diameters compared with the smallest sum occurring during the study, whereas stable disease (SD) was an insufficient shrinkage or expansion to qualify as PR or PD. Progression free survival (PFS) was calculated from the treatment initiation of the second-line therapy until PD. The OS was recorded from the paclitaxel treatment initiation until mortality, or was censored on the date of the final follow-up. Kaplan-Meier survival curves were created and compared using the log-rank test. All categorical variables were analyzed using Fisher's exact test. The Cox proportional hazards model, with stepwise regression, was applied to determine the prognostic factors for PFS at second-line treatment and OS following the start of second-line therapy, and to estimate the hazard ratios and $95 \%$ confidence intervals. $\mathrm{P}<0.05$ was considered to indicate a statistically significant difference for both one-sided and two-sided tests. All statistical analyses were performed using SAS 9.4 software (University of Massachussets, Amherst, MA, USA).

\section{Results}

Patient characteristics. The patient characteristics are shown in Table I. A total of 42 patients with metastatic urothelial bladder cancer (15 women and 27 men) received, in the period of the present study at the Jules Bordet Institute, platinum-based combination regimen as a first-line therapy and paclitaxel
Table I. Patient characteristics.

Characteristic

No. of patients

\section{Gender}

Female

Male

Median age (range), years

Treatment for cisplatin-based regimen

Pre-operative

3

Post-operative

Metastatic

Metastatic sites at the initiation of

second-line therapy

$\begin{array}{lc}\text { Lymph node } & 31 \\ \text { Lung } & 24 \\ \text { Liver } & 13 \\ \text { CNS } & 1 \\ \text { Bone } & 23\end{array}$

Biological parameters at the initiation of second-line therapy

Hemoglobin (gr/dl)

Platelets $\left(/ \mathrm{mm}^{3}\right)$

Neutrophil/lymphocyte ratio

Albumin (gr/l)

Lactate dehydrogenase (IU/l)

Creatinine (mg/dl)

Alkaline phosphatase (ALP) IU/1

single agent treatment at progression, and were included in the present analysis. The median age at diagnosis was 61-years-old. Two thirds of the cohort presented with lung metastases, $43 \%$ with other visceral involvements, notably pleural and peritoneal metastases, $29 \%$ with bone metastases, whereas liver and central nervous system involvement were more rare (Table I). All patients were treated with cisplatin-based regimens: 27 patients as a first-line chemotherapy regimen for metastatic disease, 12 patients in the post-operative setting and 3 patients in the pre-operative setting. A median progression duration of 5 months following the initial diagnosis was noted during which a median number of 4.6 cycles of cisplatin-based chemotherapy was administered on a 3 weekly basis. No CRs were observed. Following three cycles of chemotherapy, almost half of the patients achieved SD and only $20 \%$ exhibited PR. In total, $62 \%$ of the patients progressed and stopped the treatment, whereas in $16.7 \%$ of patients, the treatment was discontinued due to toxicity, predominantly cisplatin-associated renal impairment. Following the failure of the standard approach by cisplatin-based systemic chemotherapy, all patients received treatment with paclitaxel. At the initiation of this treatment, two thirds of the patients presented with lymph node involvement, $>50 \%$ with lung metastases, $42 \%$ with bone metastases and $>50 \%$ with other metastatic sites, notably pleural and peritoneal involvement, whereas only 1 patient exhibited metastatic cerebral lesions (Data not shown). From the biological point of view, 
Table II. Response to first-line platinum based regimens and second-line paclitaxel chemotherapy.

\begin{tabular}{lcc}
\hline Response & $\begin{array}{c}\text { No. patients (\%) } \\
\text { first-line cisplatin }\end{array}$ & $\begin{array}{c}\text { No. of patients (\%) } \\
\text { second line paclitaxel }\end{array}$ \\
\hline Complete response & $0(0)$ & $0(0)$ \\
Partial response & $9(21.4)$ & $4(9.5)$ \\
Stable disease & $20(47.6)$ & $15(35.7)$ \\
Progressive disease & $11(26.2)$ & $21(50.0)$ \\
Not available & $2(4.8)$ & $2(4.8)$ \\
Response rate $(\%)$ & 21.4 & 7.1 \\
Disease control rate & 69.1 & 45.2 \\
Median time to progression & 5 months & 3 months \\
Discontinuation due to progression & $26(61.9)$ & $33(78.5)$ \\
Discontinuation due to toxicities & $7(16.7)$ & $1(2.4)$ \\
\hline
\end{tabular}

Table III. Grade III/IV toxicities for patients with metastatic urothelial cancer treated with weekly paclitaxel.

\begin{tabular}{lc}
\hline Grade III/IV toxicity & No. of patients $(\%)$ \\
\hline Anemia & $3(7)$ \\
Fatigue & $1(2)$ \\
Pulmonary embolism & $8(19)$ \\
Pain due to bone metastases requiring RT & $16(38)$ \\
\hline
\end{tabular}

${ }^{\mathrm{a}} \mathrm{RT}$, radiation therapy.

it was noted that the median neutrophil to lymphocyte ratio at the initiation of paclitaxel was 7.8, almost double in comparison with the identical ratio at the initiation of cisplatin-based chemotherapy.

Treatment efficacy of second-line single agent paclitaxel following first-line cisplatin-based combination regimens. This regimen consisted of weekly intravenous administration of paclitaxel $\left(80 \mathrm{mg} / \mathrm{m}^{2}\right)$ for 3 consecutive weeks, followed by 1 week without treatment. The median duration of this regimen was 3 months. The median number of paclitaxel administrations was seven, which corresponded to the administration of 2.42 cycles. During the observation period, no patients exhibited CR, while 4,15 and 21 patients met the criteria for PR, SD and PD, respectively (Table II). Therefore, the overall response rate and disease control rate were 9.5 and $45.2 \%$, respectively. It was noted that $26.2 \%$ of patients exhibited no favorable outcome and presented with progressive disease following the first cycle of paclitaxel. The median PFS of second-line chemotherapy was 3 months and the median OS following the start of second-line therapy was 6.4 months (Table II). The PFS following second-line therapy was shorter compared with that following first-line therapy (log rank, $\mathrm{P}<0.05)$. The OS of patients since diagnosis and since initiation of paclitaxel treatment is shown in Figs. 1 and 2, respectively. The neutrophil to lymphocyte (N/1) ratio at diagnosis was not statistically correlated with the OS of the patients $(\mathrm{P}=0.96)$. At the initiation of paclitaxel, the cut-off

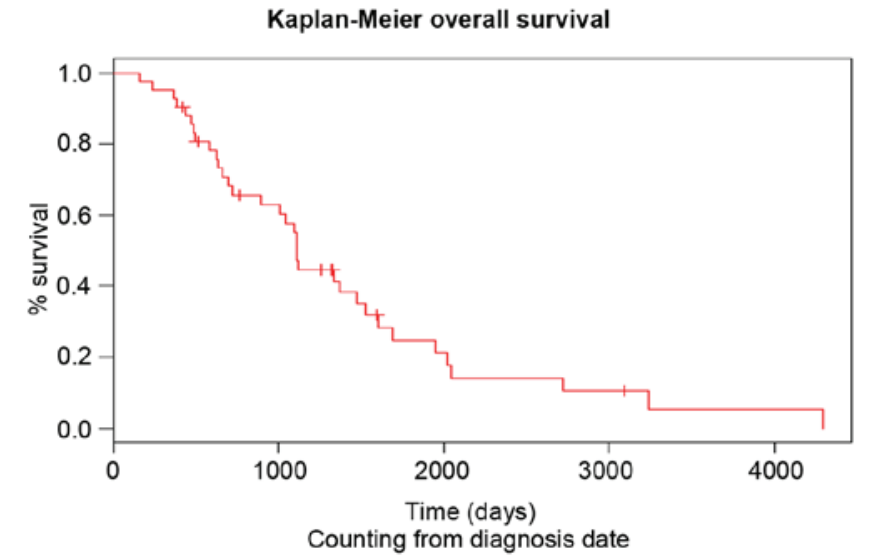

Figure 1. Overall survival of the patients with urothelial cancer since diagnosis.

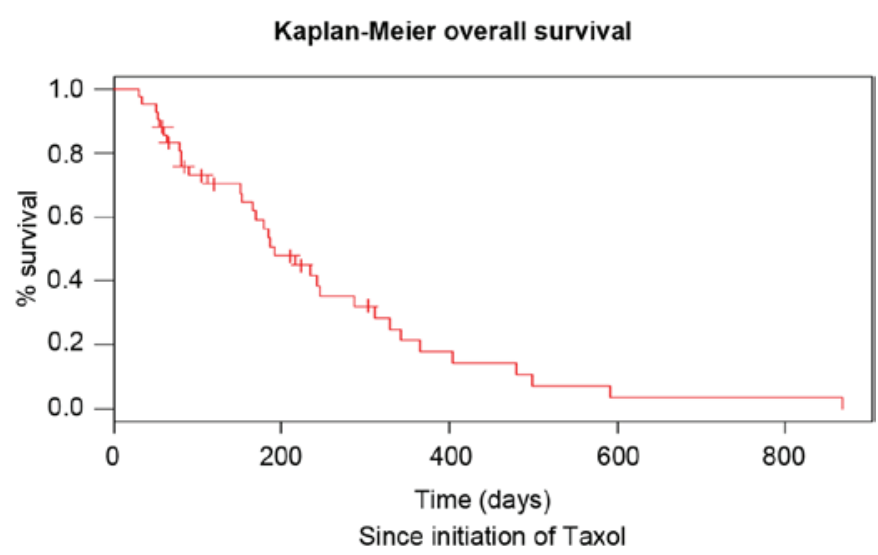

Figure 2. Overall survival of the patients with urothelial cancer since initiation of paclitaxel treatment.

value for this ratio was 4.62 and was also not prognostic for the OS ( $\mathrm{P}=0.34)$. In addition, no statistically significant difference was observed between male and female patients $(\mathrm{P}=0.722)$.

Treatment toxicity of second-line single agent paclitaxel following first-line cisplatin-based combination regimens. Weekly paclitaxel was well-tolerated. The only grade III/IV 
treatment-associated toxicities encountered were anemia and fatigue for $<10 \%$ of the population (Table III). Pulmonary embolism was detected in $19 \%$ of patients and was associated with the advanced stage of the disease compared with the treatment itself. Pain was present in $38 \%$ of patients. It was also associated with bone metastases and controlled by radiotherapy and oral analgesic treatment.

\section{Discussion}

Recurrence following first-line therapy is associated with a very poor prognosis. In this stage, management remains controversial due to the absence of randomized trials comparing second-line therapy to the optimum supportive care. Clearly, efficient well-tolerated agents suitable for palliative therapy are required. Multiple single agents have been investigated in small phase II trials, however, response rates were grim and no second-line therapy has been clearly established. Several studies have suggested that single agent paclitaxel may be active in urothelial cancer and examined its use in the second-line setting (Table IV). The first data published came from small phase II studies evaluating the response to a 3-weekly schedule of single agent paclitaxel in patients relapsing following the first-line therapy. The authors reported low efficacy with only one patient responding. Severe toxicity was non-negligible and two early mortalities occurred (26). Afterwards, it was demonstrated that weekly paclitaxel shows improved tolerance and is less toxic compared with the standard 3-weekly schedule, and therefore may provide a good second-line palliative option $(27,28)$. It is noteworthy to mention that weekly administration schedules of paclitaxel are also proven to be effective and well-tolerated in breast cancer, ovarian cancer, lung cancer and other solid tumor types $(29,30)$. The first study, which assessed the efficacy and toxicity of this schedule in patients with advanced urothelial cancer progressing following first-line therapy revealed a modest overall response rate, however a good safety profile (14). Results from a French multicenter, Groupe d'Etude des Tumeurs Uro-Génitales, phase II trial confirmed the limited objective overall response rate (31). However, the authors demonstrated disease control and clinical benefits in 47 and $24 \%$ of cases, respectively. In the present study, the efficacy of second-line paclitaxel as a single agent following first-line cisplatin-based combination regimens was also demonstrated. The disease control rate, median time to progression and median time to mortality were comparable to the previous two studies $(14,31)$. The limited overall response rate observed with paclitaxel may be partially explained by the development of resistance involving a multidrug resistance phenotype (32). The treatment was also well-tolerated with $<10 \%$ of grade III/IV treatment-associated toxicities and no mortality-associated toxicities were reported. The most common severe toxicities were fatigue and anemia.

The importance of the N/1 ratio as a prognostic factor for survival was previously elaborated in the medical literature. Cho et al (33) demonstrated that patients with ovarian cancer presenting a relative lymphopenia at diagnosis, therefore a higher N/1 ratio, exhibited worse disease outcome, probably due to a poorer lymphocyte-mediated immune response to malignancy (33). The identical observation was reported for several other malignancies, including gastric cancer (34), hepatic cancer (35) and non-small cell lung cancer (36). Gondo et al (37) were the first to report that the pre-treatment N/1 ratio is an independent prognostic factor for the survival of patients with bladder cancer, treated with radical cystectomy (37). To the best of our knowledge, no study has demonstrated the predictive value of this ratio at the initiation of paclitaxel for patients with metastatic urothelial cancer. In the present study, this ratio was not statistically correlated with the OS of these patients.

In conclusion, weekly paclitaxel treatment is a well-tolerated regimen for patients with advanced urothelial cancer who fail first-line cisplatin-based chemotherapy. Despite the fact that it offers a relatively low objective response rate, weekly paclitaxel is likely to provide non-negligible disease control and therefore should be considered a legitimate option as a second-line chemotherapeutic regimen for frail patients with advanced disease. For fit patients, clinical trials may be considered as the optimal option of second-line treatment for metastatic disease. If the above mentioned results are confirmed in a prospective randomized trial, weekly paclitaxel treatment $\left(80 \mathrm{mg} / \mathrm{m}^{2}\right)$ may be considered a legitimate treatment option, particularly for frail patients with advanced or metastatic urothelial cancer who failed platinum-based chemotherapy.

\section{References}

1. Jemal A, Bray F, Center MM, Ferlay J, Ward E and Forman D: Global cancer statistics. CA Cancer J Clin 61: 69-90, 2011.

2. Pelucchi C, Bosetti C, Negri E, Malvezzi M and La Vecchia C: Mechanisms of disease: The epidemiology of bladder cancer. Nat Clin Pract Urol 3: 327-340, 2006.

3. Oosterlinck W, Lobel B, Jakse G, Malmström PU, Stöckle M and Sternberg C: Guidelines on bladder cancer. Eur Urol 41: 105-112, 2002.

4. Loehrer PJ Sr, Einhorn LH, Elson PJ, Crawford ED, Kuebler P, Tannock I, Raghavan D, Stuart-Harris R, Sarosdy MF, Lowe BA, et al: A randomized comparison of cisplatin alone or in combination with methotrexate, vinblastine and doxorubicin in patients with metastatic urothelial carcinoma: A cooperative group study. J Clin Oncol 10: 1066-1073, 1992.

5. Sternberg CN, de Mulder PH, Schornagel JH, Théodore C, Fossa SD, van Oosterom AT, Witjes F, Spina M, van Groeningen CJ, de Balincourt C, et al: Randomized phase III trial of high-dose-intensity methotrexate, vinblastine, doxorubicin and cisplatin (MVAC) chemotherapy and recombinant human granulocyte colony-stimulating factor versus classic MVAC in advanced urothelial tract tumors: European organization for research and treatment of cancer protocol no. 30924. J Clin Oncol 19: 2638-2646, 2001.

6. Von der Maase H, Hansen SW, Roberts JT, Dogliotti L, Oliver T, Moore MJ, Bodrogi I, Albers P, Knuth A, Lippert CM, et al: Gemcitabine and cisplatin versus methotrexate, vinblastine, doxorubicin and cisplatin in advanced or metastatic bladder cancer: Results of a large, randomized, multinational, multicenter, phase III study. J Clin Oncol 18: 3068-3077, 2000.

7. Von der Maase H, Sengelov L, Roberts JT, Ricci S, Dogliotti L, Oliver T, Moore MJ, Zimmermann A and Arning M: Long-term survival results of a randomized trial comparing gemcitabine plus cisplatin, with methotrexate, vinblastine, doxorubicin, plus cisplatin in patients with bladder cancer. J Clin Oncol 23: 4602-4608, 2005.

8. Bellmunt J, von der Maase H, Mead GM, Skoneczna I, De Santis M, Daugaard G, Boehle A, Chevreau C, Paz-Ares L, Laufman LR, et al: Randomized phase III study comparing paclitaxel/cisplatin/gemcitabine and gemcitabine/cisplatin in patients with locally advanced or metastatic urothelial cancer without prior systemic therapy: EORTC intergroup study 30987. J Clin Oncol 30: 1107-1113, 2012. 
9. Dogliotti L, Cartenì G, Siena S, Bertetto O, Martoni A, Bono A, Amadori D, Onat H and Marini L: Gemcitabine plus cisplatin versus gemcitabine plus carboplatin as first-line chemotherapy in advanced transitional cell carcinoma of the urothelium: Results of a randomized phase 2 trial. Eur Urol 52: 134-141, 2007.

10. Meluch AA, Greco FA, Burris HA III, O'Rourke T, Ortega G, Steis RG, Morrissey LH, Johnson V and Hainsworth JD: Paclitaxel and gemcitabine chemotherapy for advanced transitional-cell carcinoma of the urothelial tract: A phase II trial of the Minnie pearl cancer research network. J Clin Oncol 19 3018-3024, 2001.

11. Gitlitz BJ, Baker C, Chapman Y, Allen HJ, Bosserman LD, Patel R, Sanchez JD, Shapiro RM and Figlin RA: A phase II study of gemcitabine and docetaxel therapy in patients with advanced urothelial carcinoma. Cancer 98: 1863-1869, 2003.

12. Bellmunt J, Théodore C, Demkov T, Komyakov B, Sengelov L, Daugaard G, Caty A, Carles J, Jagiello-Gruszfeld A, Karyakin $\mathrm{O}$, et al: Phase III trial of vinflunine plus best supportive care compared with best supportive care alone after a platinum-containing regimen in patients with advanced transitional cell carcinoma of the urothelial tract. J Clin Oncol 27: 4454-4461, 2009.

13. Sweeney CJ, Roth BJ, Kabbinavar FF, Vaughn DJ, Arning M, Curiel RE, Obasaju CK, Wang Y, Nicol SJ and Kaufman DS Phase II study of pemetrexed for second-line treatment of transitional cell cancer of the urothelium. J Clin Oncol 24: 3451-3457, 2006.

14. Vaughn DJ, Broome CM, Hussain M, Gutheil JC and Markowitz AB: Phase II trial of weekly paclitaxel in patients with previously treated advanced urothelial cancer. J Clin Oncol 20: 937-940, 2002

15. McCaffrey JA, Hilton S, Mazumdar M, Sadan S, Kelly WK, Scher HI and Bajorin DF: Phase II trial of docetaxel in patients with advanced or metastatic transitional-cell carcinoma. J Clin Oncol 15: 1853-1857, 1997.

16. Lorusso V, Pollera CF, Antimi M, Luporini G, Gridelli C, Frassineti GL, Oliva C, Pacini $M$ and De Lena M: A phase II study of gemcitabine in patients with transitional cell carcinoma of the urinary tract previously treated with platinum. Italian co-operative group on bladder cancer. Eur J Cancer 34: 1208-1212, 1998.

17. Pronzato P, Vigani A, Pensa F, Vanoli M, Tani F and Vaira F: Second line chemotherapy with ifosfamide as outpatient treatment for advanced bladder cancer. Am J Clin Oncol 20 : 519-521, 1997.

18. Bellmunt J, Cos J, Clèries R, Pérez M, Ribas A, Eres N, Murio JE, Margarit $C$ and Baselga J: Feasibility trial of methotrexate-paclitaxel as a second-line therapy in advanced urothelial cancer. Cancer Invest 20: 673-685, 2002.

19. Krege S, Rembrink V, Börgermann C, Otto T and Rübben H: Docetaxel and ifosfamide as second-line treatment for patients with advanced or metastatic urothelial cancer after failure of platinum chemotherapy: A phase 2 study. J Urol 165: 67-71, 2001

20. Lin CC, Hsu CH, Huang CY, Keng HY, Tsai YC, Huang KH, Cheng $\mathrm{AL}$ and $\mathrm{Pu}$ YS: Gemcitabine and ifosfamide as a second-line treatment for cisplatin-refractory metastatic urothelial carcinoma: A phase II study. Anticancer Drugs 18 487-491, 2007.

21. Soga N, Onishi T, Arima K and Sugimura Y: Paclitaxel Carboplatin chemotherapy as a second-line chemotherapy for advanced platinum resistant urothelial cancer in Japanese cases. Int J Urol 14: 828-832, 2007.
22. Suyama T, Ueda T, Fukasawa S, Imamura Y, Nakamura K, Miyasaka K, Sazuka T, Egoshi K, Nihei N, Hamano M, et al: Combination of gemcitabine and paclitaxel as second-line chemotherapy for advanced urothelial carcinoma. Jpn J Clin Oncol 39: 244-250, 2009.

23. Albers P, Park SI, Niegisch G, Fechner G, Steiner U, Lehmann J, Heimbach D, Heidenreich A, Fimmers R and Siener R; AUO Bladder Cancer Group: Randomized phase III trial of 2nd line gemcitabine and paclitaxel chemotherapy in patients with advanced bladder cancer : short-term versus prolonged treatment [German Association of Urological Oncology (AUO) trial AB 20/99]. Ann Oncol 22: 288-294, 2011.

24. Bajorin DF: Paclitaxel in the treatment of advanced urothelial cancer. Oncology (Williston Park) 14: 43-52; discussion 58, 2000.

25. Raghavan D: Progress in the chemotherapy of metastatic cancer of the urinary tract. Cancer 97 (Suppl 8): S2050-S2055, 2003.

26. Papamichael D, Gallagher CJ, Oliver RT, Johnson PW and Waxman J: Phase ii study of paclitaxel in pretreated patients with locally advanced/metastatic cancer of the bladder and ureter. Br J Cancer 75: 606-607, 1997.

27. Marchetti P, Urien S, Cappellini GA, Ronzino G and Ficorella C: Weekly administration of paclitaxel: Theoretical and clinical basis. Crit Rev Oncol Hematol 44 (Suppl): S3-S13, 2002.

28. Markman M: Management of toxicities associated with the administration of taxanes. Expert Opin Drug Saf 2: 141-146, 2003.

29. Perez EA et al: A large phase II study of paclitaxel administered as a weekly one hour infusion in patients with metastatic breast cancer. Proc Am Soc Clin Onco 18: 126a, 1999.

30. Fennely D, Aghajanian C, Shapiro F, O'Flaherty C, McKenzie M, O'Connor C, Tong W, Norton L and Spriggs D: Phase I and pharmacologic study of paclitaxel administered weekly in patients with relapsed ovarian cancer. J Clin Oncol 15: 187-192, 1997.

31. Joly F, Houédé N, Noal S, Chevreau C, Priou F, Chinet-Charrot P, Rolland F, Fléchon A, Henry-Amar M and Culine S: Do patients with advanced urothelial carcinoma benefit from weekly paclitaxel chemotherapy? A GEUG phase II study. Clin Genitourin Cancer 7: E28-E33, 2009.

32. Raghavan D and Huben R: Management of bladder cancer. Curr Probl Cancer 19: 1-64, 1995.

33. Cho H, Hur HW, Kim SW, Kim SH, Kim JH, Kim YT and Lee K: Pre-treatment neutrophil to lymphocyte ratio is elevated in epithelial ovarian cancer and predicts survival after treatment. Cancer Immunol Immunother 58: 15-23, 2009.

34. Yamanaka T, Matsumoto S, Teramukai S, Ishiwata R, Nagai Y and Fukushima M: The baseline ratio of neutrophils to lymphocytes is associated with patient prognosis in advanced gastric cancer. Oncology 73: 215-220, 2007.

35. Gomez D, Farid S, Malik HZ, Young AL, Toogood GJ, Lodge JP and Prasad KR: Preoperative neutrophil-to-lymphocyte ratio as a prognostic predictor after curative resection for hepatocellular carcinoma. World J Surg 32: 1757-1762, 2008.

36. Sarraf KM, Belcher E, Raevsky E, Nicholson AG, Goldstraw P and Lim E: Neutrophil/lymphocyte ratio and its association with survival after complete resection in non-small cell lung cancer. J Thorac Cardiovasc Surg 137: 425-428, 2009.

37. Gondo T, Nakashima J, Ohno Y, Choichiro O, Horiguchi Y, Namiki K, Yoshioka K, Ohori M, Hatano T and Tachibana M: Prognostic value of neutrophil-to-lymphocyte ratio and establishment of novel pre-operative risk stratification model in bladder cancer patients treated with radical cystectomy. Urology 79: 1085-1091, 2012. 